\title{
Plasma Membrane is Compartmentalized by a Self-Similar Cortical Actin Meshwork
}

\author{
Sanaz Sadegh, ${ }^{1}$ Jenny L. Higgins, ${ }^{2}$ Patrick C. Mannion, ${ }^{2}$ Michael M. Tamkun, ${ }^{3,4}$ and Diego Krapf ${ }^{1,2, *}$ \\ ${ }^{1}$ Department of Electrical and Computer Engineering, Colorado State University, \\ Fort Collins, Colorado 80523, USA \\ ${ }^{2}$ School of Biomedical Engineering, Colorado State University, Fort Collins, Colorado 80523, USA \\ ${ }^{3}$ Department of Biomedical Sciences, Colorado State University, Fort Collins, Colorado 80523, USA \\ ${ }^{4}$ Department of Biochemistry and Molecular Biology, Colorado State University, \\ Fort Collins, Colorado 80523, USA
}

(Received 30 November 2016; revised manuscript received 20 January 2017; published 9 March 2017)

\begin{abstract}
A broad range of membrane proteins display anomalous diffusion on the cell surface. Different methods provide evidence for obstructed subdiffusion and diffusion on a fractal space, but the underlying structure inducing anomalous diffusion has never been visualized because of experimental challenges. We addressed this problem by imaging the cortical actin at high resolution while simultaneously tracking individual membrane proteins in live mammalian cells. Our data confirm that actin introduces barriers leading to compartmentalization of the plasma membrane and that membrane proteins are transiently confined within actin fences. Furthermore, superresolution imaging shows that the cortical actin is organized into a selfsimilar meshwork. These results present a hierarchical nanoscale picture of the plasma membrane.
\end{abstract}

DOI: 10.1103/PhysRevX.7.011031

\section{INTRODUCTION}

The plasma membrane is a complex fluid where lipids and proteins continuously interact and generate signaling platforms in order to communicate with the outside world. One of the key mechanisms by which membrane molecules search reaction sites is based on lateral diffusion. Quantitative imaging methods, such as single-particle tracking [1-4], spatiotemporal image correlation spectroscopy [5], fluorescence correlation spectroscopy (FCS) [6,7], and stimulated emission depletion (STED)-FCS [8,9], show that the dynamics of proteins and lipids in the plasma membrane often deviate from normal diffusion. In particular, the mean square displacement (MSD) does not grow linearly in time as expected for Brownian motion [10-13]. This behavior suggests processes that hinder diffusion. Since the formation of protein complexes is governed by diffusion-mediated encounters, hindered diffusion plays fundamental roles in cell function.

Unveiling the underlying mechanisms leading to the observed anomalous diffusion on the cell membrane is critical to understanding cell behavior. Anomalous diffusion in the plasma membrane can be caused by macromolecular crowding [14], transient binding [15], heterogeneities [16,17], and membrane compartmentalization by the underlying cytoskeleton $[2,9,18,19]$. In recent years, it

\footnotetext{
*krapf@engr.colostate.edu
}

Published by the American Physical Society under the terms of the Creative Commons Attribution 4.0 International license. Further distribution of this work must maintain attribution to the author(s) and the published article's title, journal citation, and DOI.
Subject Areas: Biological Physics, Complex Systems

has become evident that a single mechanism cannot account for the complex dynamics observed in the plasma membrane [13]. We have shown that interactions with clathrin coated pits (CCPs) cause anomalous diffusion and ergodicity breaking $[15,20]$. However, it was observed that this process coexisted with a different anomalous diffusion mechanism attributed to diffusion within a fractal topology. Experimental evidence for the organization of the plasma membrane by the cortical actin cytoskeleton has been provided by measurements in cell blebs, spherical protrusions that lack actin cytoskeleton [21], and in the presence of actin-disrupting agents $[9,22,23]$. The picket-fence model explains these observations by postulating that the mobility of membrane-bound molecules is hindered by the actinbased cytoskeleton in close proximity to the plasma membrane, leading to transient confinement [2,24,25]. Confinement and segregation of membrane components can have important physiological consequences by allowing the formation of functional domains on the cell surface. However, in spite of the vast evidence that has accumulated over the last two decades, a direct observation of the dynamic compartmentalization of membrane proteins by underlying actin fences is challenging because of the spatial and temporal resolutions required for its visualization.

Here, we employ superresolution imaging and singleparticle tracking of membrane proteins to elucidate the compartmentalization of the plasma membrane by intracellular structures. While tracking individual voltage-gated potassium channels as described in our previous studies [15], we found that these membrane proteins exhibited anomalous diffusion on the cell surface. We now report that the anticorrelated dynamics are best modeled by obstructed diffusion instead of fractional Brownian motion, and we 
directly visualize the transient confinement of potassium channels by cortical actin in live cells. In order to characterize the cortical actin meshwork, we employ stochastic optical reconstruction microscopy (STORM) to obtain superresolution images in fixed cells. We find a noninteger fractal dimension for the actin cortex and a broad distribution of compartment sizes as expected for a self-similar structure. These observations consistently explain the anticorrelated subdiffusive motion of membrane proteins and provide new insights on the hierarchical organization of the plasma membrane.

\section{RESULTS}

\section{A. Kv1.4 and Kv2.1 ion channels undergo subdiffusion in the plasma membrane}

Voltage-gated potassium channels Kv1.4 and Kv2.1 were expressed in human embryonic kidney (HEK) cells, labeled with quantum dots (QDs) [15] and imaged using total internal reflection fluorescence (TIRF) microscopy at

50 frames/s so that individual molecules could be detected on the cell surface. Kv1.4 and 2.1 are similar in size, 654 and 853 amino acids, respectively, but share less than $20 \%$ overall amino acid identity [26]. They are placed into distinct gene subfamilies because of this low identity. They are most similar within a central core domain composed of six transmembrane alpha helices and the ion conducting pore. In contrast, they share no amino sequence identity within the cytoplasmic $\mathrm{N}$ - and $\mathrm{C}$-terminal regions; each Kv1.4 subunit has 402 cytoplasmic amino acids, while the Kv2.1 subunits have 624 . Both channels exist as homotetrameric structures, giving the functional channel 24 membrane-spanning domains and a total of either 1608 or 2496 cytoplasmic amino acids. Figure 1(a) shows representative trajectories of Kv1.4 channels. The motion of the ion channels was initially evaluated in terms of their time-averaged MSD,

$$
\overline{\delta^{2}(\Delta)}=\frac{1}{T-\Delta} \int_{0}^{T-\Delta}|\mathbf{r}(t+\Delta)-\mathbf{r}(t)|^{2} \mathrm{~d} t
$$

(a)

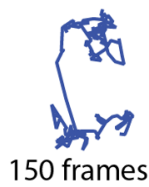

(3s)

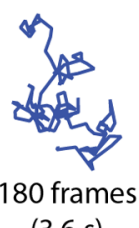

(3.6s)

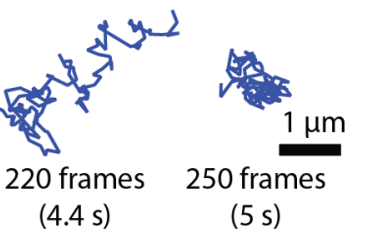

(h)

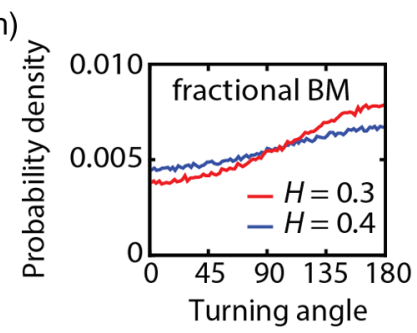

(b)

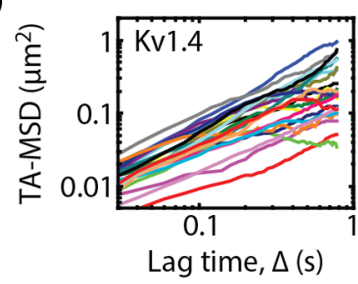

(e)

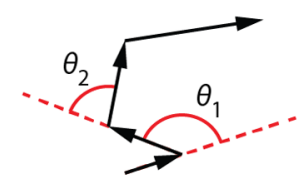

(i)

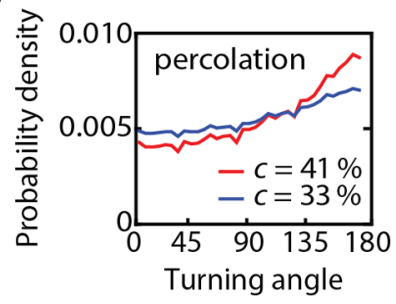

(c)

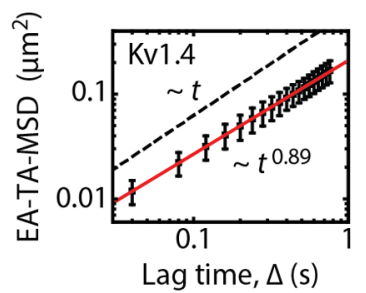

(f)

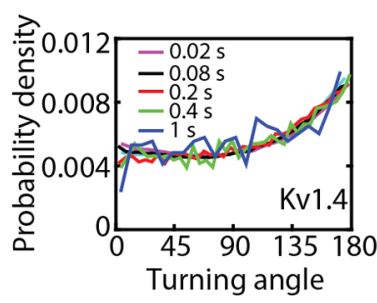

(j)

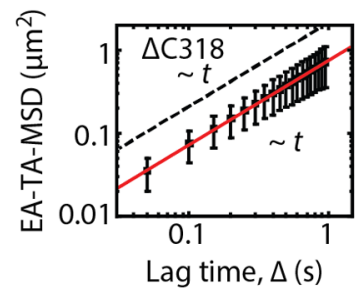

(d)

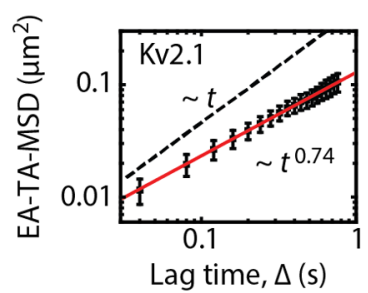

(g)

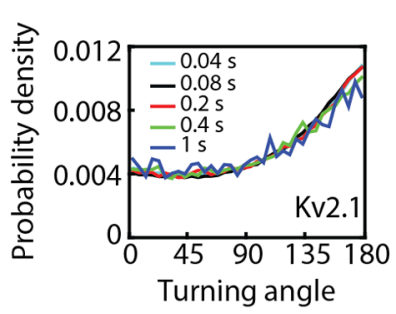

(k)

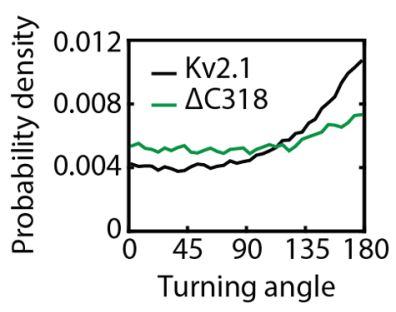

FIG. 1. Voltage-gated potassium channels Kv1.4 and Kv2.1 undergo subdiffusion in the plasma membrane. (a) Four Kv1.4 representative trajectories obtained by single-particle tracking. (b) Time-averaged MSD (TA-MSD) as a function of lag time $\Delta$ for 20 individual Kv1.4 trajectories. (c) Ensemble-averaged time-averaged MSD (EA-TA-MSD) over $1312 \mathrm{Kv} 1.4$ trajectories ( $n=10$ cells). (d) EA-TA-MSD averaged over 6,385 Kv2.1 trajectories $(n=14$ cells). The dashed lines in panels (c) and (d) are visual guides for linear behavior (free diffusion), i.e., $\left\langle\overline{\delta^{2}(\Delta)}\right\rangle \sim \Delta$. Error bars show the standard deviation. (e) Sketch illustrating the construction of turning angles from a particle trajectory. (f,g) Turning angle distributions for Kv1.4 (10 cells, 1312 trajectories) and Kv2.1 (14 cells, 6385 trajectories). Turning angle distributions are constructed for lag times between $20 \mathrm{~ms}$ and $1 \mathrm{~s}$. (h) Turning angle distributions for fractional Brownian motion simulations with Hurst exponents 0.3 and 0.4. (i) Turning angle distribution for simulations of obstructed diffusion with obstacle concentrations $33 \%$ and $41 \%$, i.e., site percolation. (j) MSD averaged over $3114 \Delta \mathrm{C} 318$ trajectories $(n=5$ cells). (k) Turning angle distributions for Kv2.1 and $\Delta \mathrm{C} 318$ (5 cells, 3114 trajectories) measured with a lag time of $200 \mathrm{~ms}$. 
where $T$ is the total experimental time, $\mathbf{r}$ the particle position, and $\Delta$ the lag time, i.e., the time difference over which the MSD is computed. When a particle displays Brownian diffusion, the MSD is linear in lag time, i.e., $\overline{\delta^{2}(\Delta)} \sim \Delta$. In contrast, anomalous diffusion is characterized by a different MSD scaling, namely, MSD $\sim \Delta^{\alpha}$, where $\alpha$ is the anomalous exponent. Anomalous diffusion is classified as subdiffusion when $0<\alpha<1$ and superdiffusion when $\alpha>1$. Figure 1(b) shows the MSD of 20 individual trajectories. The MSDs of Kv1.4 as well as Kv2.1 channels show subdiffusive behavior, albeit with large apparent fluctuations. Figures 1(c) and 1(d) show the MSDs averaged over $1312 \mathrm{Kv} 1.4(n=10$ cells $)$ and 6385 Kv2.1 $\left(n=14\right.$ cells) trajectories, $\left\langle\overline{\delta^{2}(\Delta)}\right\rangle$, respectively. Throughout the paper, we employ overlines to denote time averages and brackets to denote ensemble averages. The anomalous exponent $\alpha$ of Kv1.4 was found to be 0.89 and that of Kv2.1 was 0.74 , indicating subdiffusion in both cases.

Several distinct mathematical models lead to subdiffusion [11-13]. Among the most well-accepted types of subdiffusion in biological systems, we encounter (i) obstructed diffusion, (ii) fractional Brownian motion (fBM), and (iii) continuous time random walks (CTRW). Both fBM [27,28] and obstructed diffusion [29-31] are models for subdiffusive random walks with anticorrelated increments that have been extensively used in live cells. Note that fBM describes the motion in a viscoelastic fluid $[32,33]$, which can be caused by macromolecular crowding [34,35]. fBM is a generalization of Brownian motion that incorporates correlations with power-law memory. It is characterized by a Hurst exponent $H$ that translates into an anomalous exponent $\alpha=2 H$. Obstructed diffusion describes the motion of a particle hindered by immobile (or slowly moving) obstacles, e.g., percolation. As the concentration of immobile obstacles increases, the availability of space decreases. Near a critical concentration known as the percolation threshold, the obstacles form a fractal with dead ends in all length scales. In particular, the reduction of the available space results in anomalous diffusion with a recurrent exploration pattern. A CTRW is a generalization of a random walk where a particle waits for a random time between steps [36]. When the waiting times are asymptotically distributed according to a power law such that the mean waiting time diverges, the CTRW is subdiffusive. These three models describe very distinct physical underlying mechanisms, but they can yield similar sublinear MSD scaling, particularly in obstructed diffusion and fBM models. Thus, the MSD analysis is insufficient to elucidate the type of random walk.

Different tests beyond the MSD have been employed to distinguish between types of subdiffusive random walks, including $p$ variations [37], first-passage probability distribution [38], mean maximal excursion [39], Gaussianity [40], and fractal dimensions [41]. Here, we employ the distribution of directional changes, i.e., the turning angles, a tool that probes correlations in the particle displacements and has been shown to contain information on the complexity of a random walk [42]. Figure 1(e) illustrates the construction of turning angles from a particle trajectory. In simple Brownian motion, the turning angles are uniformly distributed. Contrastingly, when the steps are correlated, the distribution of turning angles is not uniform [42]. Figures 1(f) and 1(g) show the distribution of turning angles of Kv1.4 and Kv2.1 for different lag times (1312 Kv1.4 tracks, 10 cells and $6385 \mathrm{Kv} 2.1$ tracks, 14 cells). Both distributions peak at $\theta=180^{\circ}$, indicating that the particles are more likely to turn back than to move forward. In other words, $\mathrm{Kv}$ channels have a preference to go back in the direction from where they came rather than to persist moving in the same direction. This property is a fingerprint of subdiffusive random walks with anticorrelated increments. Besides the shape of the distribution, the dependence on lag time bears valuable information. Strikingly, we observe that the distribution is independent of lag time; i.e., we measure the same distribution of directional changes whether the lag time is $20 \mathrm{~ms}$ or $1 \mathrm{~s}$.

We examined numerical simulations of fBM and obstructed diffusion and found that they have distinctive attributes in their distribution of directional changes. Figure 1(h) shows the distribution of directional changes for subdiffusive fBM simulations with Hurst exponents $H=0.3$ and 0.4. Even though the distributions peak at $180^{\circ}$, the probability density function is different from the experimental data [Figs. 1(f) and $1(\mathrm{~g})]$. In our experimental data, the turning angle distributions increase sharply as $\theta$ approaches $180^{\circ}$, and most of the deviations from a uniform distribution are above $90^{\circ}$. However, fBM gives rise to a gradual increase that takes place mainly in the range $45^{\circ}<\theta<135^{\circ}$. Furthermore, the turning angles of fBM reach a plateau, in contrast to our measurements. Conversely, obstructed diffusion strongly resembles our experimental results. Figure 1(i) shows the turning angle distribution for obstructed diffusion simulations in a square lattice with obstacle concentrations $33 \%$ and $41 \%$ [31]. Note that $41 \%$ is slightly above the percolation threshold. These results show that the motion of $\mathrm{Kv}$ channels in the plasma membrane is better modeled by percolation, i.e., obstructed diffusion, rather than motion in a viscoelastic medium, i.e., fBM.

Potential obstacle candidates for obstructed diffusion in the plasma membrane are the cortical cytoskeleton, lipid rafts, and extracellular glycans. By evaluating the MSD and turning angle distribution of $\Delta \mathrm{C} 318$, a mutant in which the last 318 amino acids of the C-terminus of the Kv2.1 channel had been deleted [43], we found that the anticorrelated diffusion originates from interactions with intracellular structures. We observed that $\Delta \mathrm{C} 318$ channels diffuse freely in the plasma membrane, $\alpha=1$, with a diffusion coefficient $D=0.19 \mu \mathrm{m}^{2} / \mathrm{s}$ [Fig. 1(j), $n=3114$ tracks, 5 cells]. Furthermore, the distribution of turning angles of $\Delta \mathrm{C} 318$ 
was flattened, as expected for Brownian diffusion [Fig. 1(k)], indicating that the intracellular C-terminal domain of $\mathrm{Kv} 2.1$ plays a key role in the anticorrelations within the particle trajectory. Even though the distribution of turning angles in the $\Delta \mathrm{C} 318$ mutant is close to that in Brownian motion, a small peak is still noticeable at $180^{\circ}$, suggesting additional complexities in the plasma membrane.

In contrast to Kv1.4, which is homogeneously distributed on the cell membrane, a subpopulation of Kv2.1 channels forms micron-sized clusters that localize to endoplasmic reticulum (ER)-plasma membrane junctions $[44,45]$. Thus, we expect that both the ER and the cortical cytoskeleton introduce intracellular interactions with Kv2.1 channels. To identify the origin of the observed anticorrelated diffusion, we analyzed the motion of nonclustered Kv2.1 channels, i.e., the channels that reside outside ER-plasma membrane junctions. We labeled Kv2.1 channels both with green fluorescent protein (GFP) and QDs [45]. While all the channels were labeled with GFP, only a small fraction included QDs in order to enable both single-particle tracking and cluster identification (Ref. [46], Fig. S1). We observed that the distribution of directional changes of nonclustered channels is indistinguishable from that of the overall population (Ref. [46], Fig. S2). Thus, we can exclude interactions with the ER as the cause for anticorrelated subdiffusion. These observations suggest that diffusion is hindered by intracellular components, possibly the cortical cytoskeleton, in agreement with a membrane-skeleton fence model [2].

We observed that the distribution of turning angles was independent of lag times [Figs. 1(f) and 1(g)] within the probed spatial and temporal scales. These observations indicated that the anticorrelated subdiffusion of Kv channels did not have an evident characteristic time scale. This type of random walk is consistent with diffusion on a self-similar structure, i.e., a fractal subspace. In order to visualize the difference between diffusion on a fractal structure and diffusion on a meshwork with a characteristic length scale, we performed simulations of motion of a particle in the presence of permeable fences that introduce compartments with a well-defined length scale (Ref. [46], Fig. S3). In these simulations, we observed that the distribution of turning angles is not time invariant; the peak at $180^{\circ}$ grows as we increase the lag time up to a characteristic time, and then it decays when the lag time increases further [Ref. [46], Figs. S3(b) and S3(c)]. Thus, hop diffusion with a narrow distribution of confinement sizes exhibits a time-dependent turning angle distribution (with a well-defined characteristic time scale), in contrast to our experimental results where the turning angle distribution is time invariant.

\section{B. Cortical actin transiently confines Kv1.4 and Kv2.1 channels}

We observed that $\mathrm{Kv}$ channels undergo obstructed diffusion. The $\Delta \mathrm{C} 318$ mutant data indicated that hindering of the particle motion originated within cytoplasmic structures in close proximity to the plasma membrane, in agreement with previous experimental evidence of transient confinement by the actin-based cytoskeleton [9,21,47-49]. Thus, we examined the cortical actin as a candidate for the observed obstructed diffusion in the plasma membrane.

We imaged the cortical actin in live HEK cells using the photoactivatable probe tdEosFP [50] via an actin-binding peptide (ABP) that reversibly binds to F-actin [51]. Previous studies showed that expression of ABP-tdEosFP does not affect the organization of the cytoskeleton [51,52]. By activating a sparse subset of tdEosFP and individually localizing them with high precision, we generated photoactivated localization microscopy (PALM) images using localizations from 100 frames ( $2 \mathrm{~s})$, yielding a smooth video of the dynamic actin meshwork (Ref. [46], Video S1). Both excitation and photoactivation were implemented in total internal reflection fluorescence (TIRF) so that only the actin adjacent to the plasma membrane was imaged. The dissociation of ABP-tdEosFP occurs with a time constant on the order of $40 \mathrm{~s} \mathrm{[51];} \mathrm{thus,} \mathrm{the} \mathrm{exchange} \mathrm{within} \mathrm{2-s} \mathrm{imaging} \mathrm{is}$ negligible.

Figure 2(a) shows a representative PALM reconstruction of actin. Although the number of localizations in 100 frames is not adequate to fully resolve the cortical actin and some faint fluorescent single-filament structures might be missed in the images, we could use the reconstructed PALM image to study the interactions of the potassium channels with the actin cortex in live cells. Previous breakthrough experiments have reported simultaneous imaging of cortical actin cytoskeleton and single-particle tracking $[49,53,54]$. Here, to the best of our knowledge, we perform, for the first time, simultaneous single-particle tracking measurements and imaging cortical actin with superresolution.

In order to find out whether actin-delimited domains as identified by PALM hinder diffusion and compartmentalize the cell surface, we imaged and tracked Kv1.4 and Kv2.1 channels on the cell surface while simultaneously imaging the cortical actin. Channels often remained confined within the areas enclosed by actin, indicating that actin acted as a barrier to channel diffusion. Figures 2(a) and 2(b) show Kv2.1 channel tracks for one video overlaid on the last reconstructed image of the cortical actin. However, this visualization method suffers from overlaying long trajectories on a single reconstruction image of the actin meshwork. In addition to being constrained by actin structures, some trajectories exhibit confinement within small nanoscale domains that do not appear to be enclosed by actin. We have previously shown that $\mathrm{Kv}$ channels exhibit frequent immobilizations when the channels are captured within clathrin-coated pits [20]. Thus, the cortical actin cytoskeleton is not the sole mechanism by which the mobility of $\mathrm{Kv}$ channels is hindered. In order to deal with 
(a)

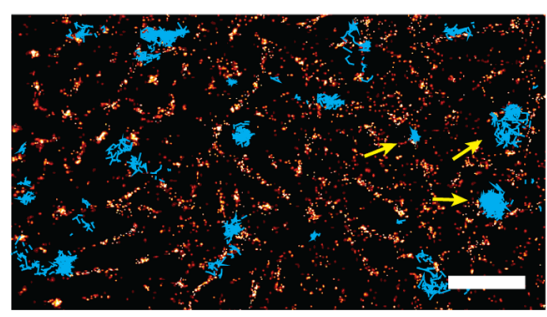

(c)

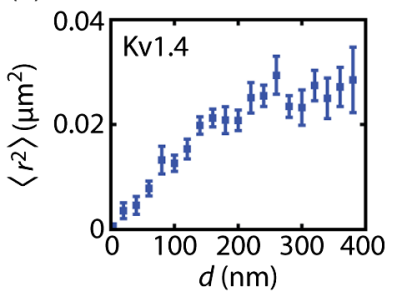

(b)
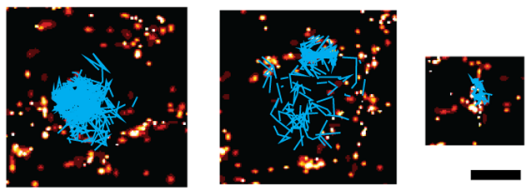

(e)

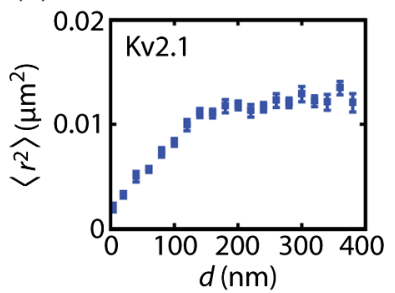

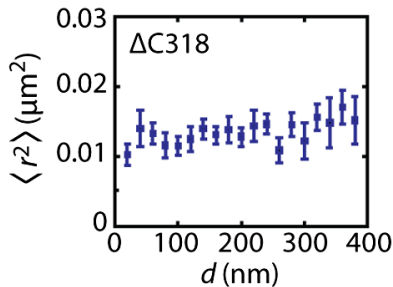

FIG. 2. Cortical actin transiently confines Kv channels. (a) Trajectories of individual Kv2.1 channels (shown in cyan) overlaid on an actin PALM image (shown in red). The scale bar is $2 \mu \mathrm{m}$. (b) Enlargements of the areas indicated with yellow arrows in panel (a). The scale bar is $500 \mathrm{~nm}$. The left trajectory shows confinement in a large compartment, the middle one shows hopping between two compartments, and the right one shows confinement in a nanoscale domain. (c)-(e) Mean-square displacements $\left\langle r^{2}\right\rangle$ covered by Kv1.4 and Kv2.1 and $\Delta$ C318 channels in $200 \mathrm{~ms}$ as a function of their maximum distance from the nearest actin feature. Error bars indicate standard errors.

these complexities, we evaluated the MSDs as a function of proximity to actin.

Given that actin hinders channel motility, we expect the particles to explore smaller areas when they are confined within smaller compartments. To test the actin-fence hypothesis, we overlaid channel trajectories on the corresponding PALM image of actin obtained in $2 \mathrm{~s}$, a time scale in which the actin structure is fairly persistent (Ref. [46], Video 1), with a sliding time window of $0.2 \mathrm{~s}$. For example, trajectories of the channels from 0 to $2 \mathrm{~s}$ were overlaid on the first reconstructed actin frame, and the trajectories in the interval $200 \mathrm{~ms}$ to $2.2 \mathrm{~s}$ were overlaid on the second actin PALM frame. Then, we partitioned the trajectories into 200-ms intervals and classified each segment according to the maximum distance $d$ of the particle to the nearest actin feature, calculated using a Euclidean distance map algorithm. We evaluated the ensemble-averaged MSD $\left\langle r^{2}\right\rangle$ of all the segments located at a specific distance away from actin; i.e., we averaged the squared displacements in $200 \mathrm{~ms}$ of the particles transiently located a given distance from actin. Figures 2(c) and 2(d) show the MSD as a function of distance to actin for Kv1.4 and Kv2.1. For both channels, we observed that, as molecules dwell closer to actin, their MSD decreases.

As a control of our method, we performed the same analysis for $\Delta \mathrm{C} 318$ channels. Because of the lack of the intracellular domain, these channels should not have any interaction with the cortical cytoskeleton. We observed that the MSD of $\Delta \mathrm{C} 318$ channels is independent of distance from actin [Fig. 2(e)], which demonstrates the effect of intracellular structure in the transient confinement of Kv channels.

\section{Characterization of cortical actin meshwork}

When imaging live cells with PALM, the number of frames used in the reconstruction is restricted by the dynamic nature of the cells. A low number of frames results in insufficient detected particles to accurately determine the structure, also having a deleterious effect on resolution. In contrast, very high spatial resolution can be obtained in fixed cells by collecting data over long times [55-57]. Therefore, we used TIRF-STORM to visualize the compartments formed by cortical actin in fixed cells. Actin was labeled with phalloidin conjugated to Alexa Fluor 647, which binds actin filaments with high specificity without significantly enlarging them [57]. A total of 50000 frames were used in the reconstruction. In our STORM reconstructions, we observed both thick and thin actin structures, Fig. 3(a). The finest structures that we observed had a cross-section standard deviation of $20 \mathrm{~nm}(\mathrm{FWHM}=48 \mathrm{~nm})$. Figure 3(b) shows the average cross-section profile of 20 lines aligned by the center of each line. The thickness of these lines in the reconstruction is governed by the localization accuracy, $20 \pm 8 \mathrm{~nm}$ (mean \pm SD; Ref. [46], Fig. S4), which sets a lower bound on STORM resolution. Thus, we are unable to determine whether these structures are individual filaments (10 $\mathrm{nm}$ in diameter) or actin bundles.

We employed a watershed segmentation algorithm [58] to identify actin-delimited compartments in the STORM reconstructions [Figs. 3(c) and 3(d)] across the whole cell. The average percentage of the watershed meshwork covered by actin was $84 \pm 4 \%$ (mean $\pm \mathrm{SD}, n=9$ cells). Figure 3(e) shows the distribution of compartment areas $(n=2500$ compartments). The areas of the compartments are fitted 
(a)

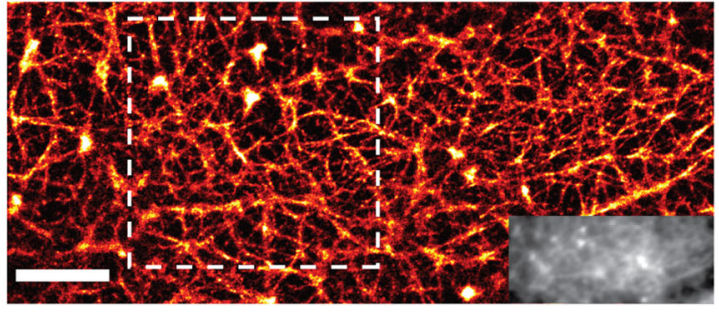

(c)

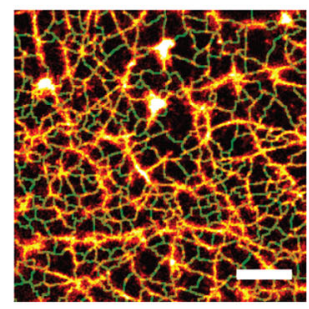

(d)

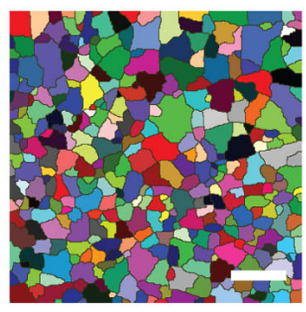

(b)
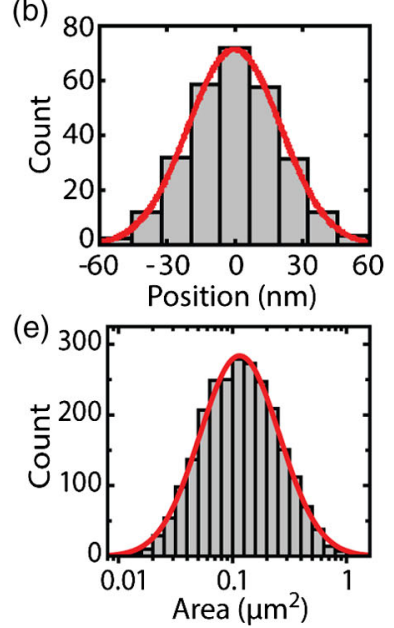

FIG. 3. Characterizations of actin compartments. (a) Superresolution STORM image of the cortical actin in a HEK cell. The inset shows the conventional TIRF image. The scale bar is $2 \mu \mathrm{m}$. (b) Average cross-section profile of 20 filaments aligned by the center of each line. The red line is a Gaussian fit with standard deviation $\sigma=20 \mathrm{~nm}$. (c) Watershed segmentation (shown in green) of the boxed area overlaid on the STORM image. (d) Compartments determined by watershed are designated with different colors. Scale bars in panels (c) and (d) are $1 \mu \mathrm{m}$. (e) Distribution of compartment areas for fixed cells ( 9 cells, $n=2500$ compartments). Areas are shown in logarithmic scale, and the red line is a log-normal distribution.

well by a log-normal distribution, which is a subexponential heavy-tailed distribution in the sense that it decays more slowly than any exponential tail [59]. The log-normal distribution is in good agreement with Kolmogorov's model for the distribution of particle sizes after repeated breakage $[60,61]$. When a particle is divided into fragments in such a way that the fragment proportions are independent of the original particle size, a log-normal distribution emerges in the particle sizes after random repeated fragmentation. Analogously, actin-delimited compartments are split into smaller compartments by growing actin filaments; thus, their distribution is predicted to be log normal.

In addition to the compartment area distribution, the relation between perimeter and area contains valuable information. Perimeter-area relations have been extensively used to investigate the properties of complex planar shapes $[62,63]$. As expected, we observe that areas and perimeters of the different compartments are highly correlated [Fig. 4(a)]. This correlation indicates shape homogeneity among different compartments [64]. Furthermore, the area exhibits the same scaling over the whole observed range, $A \sim L^{1.8}$, where $A$ and $L$ are compartment area and perimeter, indicating the same statistical character at different scales and suggesting that compartments formed by cortical actin are scale invariant in the observed range. Such scale invariance is a hallmark of self-similar fractal structures.

Fractals are characterized by scaling properties governed by a noninteger dimension $d_{f}$, i.e., an anomalous dependence of the "mass" on the linear size of the system with $M \sim l^{d_{f}}$. In a regular object such as a line, square, or cube, we would refer to its mass $M$ as the length, area, or volume, respectively. In these regular cases, the mass scales as
$M \sim l^{d}$, where $l$ is the linear size and $d=1,2,3$ is the spatial dimension. On the other hand, fractals such as a Sierpinsky gasket or a percolation cluster differ from Euclidean spaces and display a fractional dimension $[62,65]$. Usually, the capacity dimension is obtained using a box-counting algorithm that quantifies the mass scaling. In brief, the structure is placed on a grid, the number of occupied "boxes" are counted, and the process is iterated for finer grids. The number of occupied boxes scales as $N \sim \epsilon^{-d_{f}}$, where $\epsilon$ is the box length. Figure 4(b) shows the computation of the fractal dimension of the cortical actin meshwork from the STORM image in a representative cell. The box-counting analysis shows that the actin structure exhibits statistical self-similarity over more than three decades. Our data indicate that the fractal dimension of the meshwork is $d_{f}=1.75 \pm 0.02(n=9$ cells $)$.
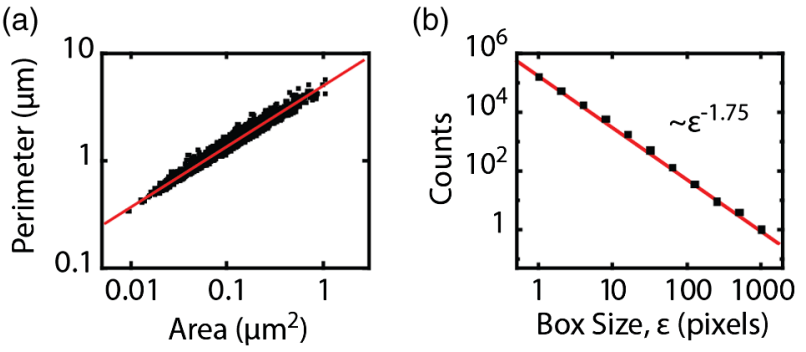

FIG. 4. Fractality of the cortical actin meshwork. (a) Log-log scatter plot of compartment perimeter vs compartment area. The fitted line corresponds to $L=4.8 A^{0.55}$ (Pearson correlation coefficient $\rho=0.98$ in $\log$ scales). (b) Representative example of box-counting algorithm in one cell where the exponent yields $d_{f}=1.75$. 


\section{DISCUSSION}

Our current understanding of the plasma membrane is that of a complex partitioned fluid where molecules often undergo anomalous diffusion and can be segregated according to their function. We observe that $K^{+}$channels perform a random walk with antipersistent nature, i.e., a random walk with an increased probability of returning to the site it just left. However, elucidating the mechanisms that cause anomalous diffusion is not trivial because several different subdiffusion models lead to similar MSD scaling. The analysis of $K^{+}$channel motion is further complicated by the occurrence of immobilizations with power-law sojourn times, which introduce deviations from Gaussian functions in the distribution of displacements [15,20]. Thus, we cannot employ Gaussianity-based tests to distinguish among complex antipersistent random walks. We find that the distribution of directional changes provides a robust test for the type of random walk. The measured channel trajectories are shown to be well described by obstructed diffusion but not by fBM.

We observed that the Kv2.1 intracellular domain played a key role in the anomalous diffusion, in agreement with previous observations showing that the depth at which a membrane protein extends into the cytoplasm determined how frequently it encountered mechanical barriers [48]. The obvious candidate to obstruct the motion of proteins with large intracellular domains is the actin cytoskeleton. Thus, we visualized the cortical actin with high temporal and spatial resolution and evaluated its effect on membrane protein dynamics. Considering that some faint singlefilament actin structures might not be accurately detected by PALM imaging, we can miss some interactions between actin and membrane proteins. Notwithstanding, we found that $\mathrm{Kv}$ channels are transiently confined by permeable actin fences, confirming existing models for membrane compartmentalization as an organizing principle of the actin cytoskeleton [2]. By studying the diffusion of Kv2.1 channels outside ER-plasma membrane junctions, we verified that the observed subdiffusion is not due to interactions with ER. Other intracellular components such as intermediate filaments could also hinder protein diffusion and further compartmentalize the cell membrane, but these cytoskeletal filaments were not studied in the present work. Previous single-particle tracking studies using lipids labeled with 40-nm gold nanoparticles have observed the compartmentalization of the plasma membrane of HEK293 cells, with a 70-nm mean compartment size [1]. However, this compartmentalization occurs with molecules having virtually no cytoplasmic domains and with a residence time close to $3 \mathrm{~ms}$. At time scales above $50 \mathrm{~ms}$, gold-labeled lipids were found to exhibit Brownian diffusion with an effective diffusion coefficient $D=0.41 \mu \mathrm{m}^{2} / \mathrm{s}$ [1], similar to our observations for the $\Delta \mathrm{C} 318 \mathrm{Kv} 2.1$ mutant.

Ion channels are observed to undergo anticorrelated anomalous diffusion over at least 2 orders of magnitude in time. In terms of percolation theory, this hints that the cell surface is maintained close to criticality, i.e., near the percolation threshold. However, this hypothesis seems highly unlikely. A more feasible explanation stems from the emergence of a scale-invariant structure under the plasma membrane. We directly observed that, within the probed spatial scale, the actin cortex in fact has a selfsimilar nature. It is possible to speculate that actin fractality develops from its branching structure. Hierarchically branched structures have a fractal dimension $d_{f}$ such that $R_{b}=R_{r}^{d_{f}}$, where $R_{b}$ is the bifurcation ratio and $R_{r}$ is the length-order ratio [66]. The bifurcation ratio can be interpreted as the average number of branches that emerge after a bifurcation, and the length-order ratio is defined as the ratio between incoming branch length and the length of the emerging branches until the next bifurcation [67]. Actin branching is driven by the Arp2/3 complex [68] with a bifurcation ratio $R_{b}=2$. Here, we measure a meshwork fractal dimension $d_{f}=1.75$, which can arise from a branching pattern with $R_{r}=1.5$ or, in other words, the daughter branch being, on average, $1 / 3$ shorter than the mother branch. The fractal dimension of the cytoskeleton is in line with a broad range of fractal geometries found in biology, ranging from the lung alveoli to subcellular structures such as mitochondrial membranes and the endoplasmic reticulum [62].

We propose that the fractal nature of the actin cortex is employed by the cell to organize the plasma membrane. The complexity of this structure leads to a hierarchical organization with domains in multiple length scales and the development of nested compartments. Such a dynamic hierarchical organization facilitates the active segregation of domains with different functions and the maintenance of reactants near reaction centers. Furthermore, the fractal nature of the cortical actin has broad implications for anomalous diffusion; for instance, it could bridge the gap between the plasma-membrane hop-diffusion models and diffusion in a fractal that leads to anomalous dynamics over broad time scales. We foresee that a self-similar cytoskeleton structure also influences active actomyosin-mediated organization of the plasma membrane [69] in such a way that these processes can take place over multiple length scales.

In conclusion, our findings show that the plasma membrane is compartmentalized in a hierarchical fashion by a dynamic cortical actin fractal. We find that the anomalous diffusion of potassium channels is best modeled by obstructed diffusion or diffusion in a fractal. By combining PALM imaging with single-particle tracking, we were able to directly visualize the hindering effect of cortical actin on the diffusion of the membrane proteins. We characterized the compartments formed by cortical actin using superresolution imaging in fixed cells and found evidence for the self-similar topology of this structure. 


\section{MATERIALS AND METHODS}

\section{A. Cell transfection and labeling}

HEK 293 cells (passage 42-49; American Type Culture Collection) were cultured in phenol red Dulbecco's Modified Eagle's Medium (DMEM), supplemented with $10 \%$ fetal bovine serum (FBS; Gibco) at $37^{\circ} \mathrm{C}$. Cells were transfected to express a Kv2.1 or Kv1.4 construct with an extracellular biotin acceptor domain that, when coexpressed with a bacterial biotin ligase, results in biotinylated $\mathrm{Kv}$ channels on the cell surface [70]. For live-cell actin imaging, cells were transfected with $3 \mu \mathrm{g}$ of ABP-tdEos. $\mathrm{ABP}$ is the actin-binding sequence of ABP140 from $S$. cerevisiae, consisting of 17 amino acids [51,52]. Kv2.1-loopBAD-GFP (3 $\mu \mathrm{g})$ was employed to identify Kv2.1 clusters on the cell surface.

For single-particle tracking, biotinylated channels were labeled with QDs. Cells were incubated for 10 minutes in HEK imaging saline with $1 \mathrm{nM}$ streptavidin-conjugated QD705 or QD655 (Invitrogen) and $10 \mathrm{mg} / \mathrm{mL}$ bovine serum albumin (BSA) [15] at $37^{\circ} \mathrm{C}$. Following incubation, the cells were rinsed again six times with HEK imaging saline to ensure the removal of any unbound QDs. The diameter of the QDs is in the range 10-15 nm; thus, given that $\mathrm{Kv}$ channels are similar in size to the QDs, it is highly unlikely that the Kv:QD stoichiometry is higher than 1:1. We have previously shown that QD conjugation does not alter Kv channel diffusion [15,20].

\section{B. Live cell imaging}

Imaging was performed in an objective-based TIRF microscope built around an IX71 Olympus body. The sample temperature was kept at $37^{\circ} \mathrm{C}$ using an objective and stage heater (Bioptechs). A 405-nm laser was used to activate tdEosFP fluorophore, while 473-nm and 561-nm lasers were used to excite it in its inactive and active states, respectively.

\section{Single-particle tracking}

QD labeling was controlled so that QDs remained at low density to allow for single-particle tracking [15]. Particle detection and tracking were performed in MATLAB using u-track [71].

\section{Fixed cell imaging}

Cells were plated on Matrigel-coated 35-mm Petri dishes. After 12 hours, the cells were fixed and labeled with Alexa Fluor 647-phalloidin (Invitrogen). Image stacks were obtained using the same setup as live cell imaging. A continuous illumination of a 638-nm laser was used to excite the Alexa Fluor 647. The laser power before the objective was $30 \mathrm{~mW}$. To maintain an appropriate density of activated molecules, a 405-nm laser was used in some experiments. To generate a superresolution image, 50000 frames were collected.

\section{E. Fractal dimension}

The fractal dimension of the actin cortex was computed using a box-counting algorithm. The thresholded binary actin image was placed on a grid of square boxes of size $\epsilon$, and the number of occupied boxes was counted. The process was repeated for grids of boxes with different sizes. The number of occupied boxes scales as

$$
N \sim \epsilon^{-d_{f}},
$$

where $d_{f}$ is the capacity dimension, or simply the fractal dimension.

\section{F. Statistics}

Results show mean and s.e.m. unless indicated otherwise. All experimental results were obtained from multiple different dishes and days. The number of distinct imaging regions, i.e., different cells, is indicated in the text.

\section{ACKNOWLEDGMENTS}

We thank Keith Lidke and Sheng Liu for the codes for PALM reconstruction, Maxime Dahan for providing the plasmids to express ABP-tdEos, and Aubrey Weigel, Liz Akin, María Gracia Gervasi, Phil Fox, Ben Johnson, and Xinran $\mathrm{Xu}$ for useful discussions. We gratefully acknowledge the support of NVIDIA Corporation with the donation of a GeForce GTX TITAN used for this research. D. K. and J.L.H. thank Nikki Curthoys for technical advice with superresolution microscopy during the initial stages of the project. This work was supported by the National Science Foundation under Grant No. 1401432 (to D. K.) and the National Institutes of Health under Grant No. R01GM109888 (to M. M. T.).

[1] K. Murase, T. Fujiwara, Y. Umemura, K. Suzuki, R. Iino, H. Yamashita, M. Saito, H. Murakoshi, K. Ritchie, and A. Kusumi, Ultrafine Membrane Compartments for Molecular Diffusion as Revealed by Single Molecule Techniques, Biophys. J. 86, 4075 (2004).

[2] A. Kusumi, C. Nakada, K. Ritchie, K. Murase, K. Suzuki, H. Murakoshi, R. S. Kasai, J. Kondo, and T. Fujiwara, Paradigm Shift of the Plasma Membrane Concept from the Two-Dimensional Continuum Fluid to the Partitioned Fluid: High-Speed Single-Molecule Tracking of Membrane Molecules, Annu. Rev. Biophys. Biomol. Struct. 34, 351 (2005).

[3] S. Manley, J. M. Gillette, G. H. Patterson, H. Shroff, H. F. Hess, E. Betzig, and J. Lippincott-Schwartz, High-Density Mapping of Single-Molecule Trajectories with Photoactivated Localization Microscopy, Nat. Methods 5, 155 (2008).

[4] M. P. Clausen and B. Christoffer Lagerholm, Visualization of Plasma Membrane Compartmentalization by High-Speed Quantum Dot Tracking, Nano Lett. 13, 2332 (2013). 
[5] C. Di Rienzo, E. Gratton, F. Beltram, and F. Cardarelli, Fast Spatiotemporal Correlation Spectroscopy to Determine Protein Lateral Diffusion Laws in Live Cell Membranes, Proc. Natl. Acad. Sci. U.S.A. 110, 12307 (2013).

[6] P.-F. Lenne, L. Wawrezinieck, F. Conchonaud, O. Wurtz, A. Boned, X.-J. Guo, H. Rigneault, H.-T. He, and D. Marguet, Dynamic Molecular Confinement in the Plasma Membrane by Microdomains and the Cytoskeleton Meshwork, EMBO J. 25, 3245 (2006).

[7] V. Ruprecht, S. Wieser, D. Marguet, and G. J. Schütz, Spot Variation Fluorescence Correlation Spectroscopy Allows for Superresolution Chronoscopy of Confinement Times in Membranes, Biophys. J. 100, 2839 (2011).

[8] A. Honigmann, V. Mueller, H. Ta, A. Schoenle, E. Sezgin, S. W. Hell, and C. Eggeling, Scanning STED-FCS Reveals Spatiotemporal Heterogeneity of Lipid Interaction in the Plasma Membrane of Living Cells, Nat. Commun. 5, 5412 (2014).

[9] D. M. Andrade, M. P. Clausen, J. Keller, V. Mueller, C. Wu, J. E. Bear, S. W. Hell, B. Christoffer Lagerholm, and C. Eggeling, Cortical Actin Networks Induce Spatio-temporal Confinement of Phospholipids in the Plasma Membrane-A Minimally Invasive Investigation by STED-FCS, Sci. Rep. 5, 11454 (2015).

[10] E. Barkai, Y. Garini, and R. Metzler, Strange Kinetics of Single Molecules in Living Cells, Phys. Today 65, No. 08, 29 (2012).

[11] F. Höfling and T. Franosch, Anomalous Transport in the Crowded World of Biological Cells, Rep. Prog. Phys. 76, 046602 (2013).

[12] R. Metzler, J.-H. Jeon, A. G. Cherstvy, and E. Barkai, Anomalous Diffusion Models and Their Properties: Nonstationarity, Non-ergodicity, and Ageing at the Centenary of Single Particle Tracking, Phys. Chem. Chem. Phys. 16, 24128 (2014).

[13] D. Krapf, Mechanisms Underlying Anomalous Diffusion in the Plasma Membrane, Curr. Top. Membr. 75, 167 (2015).

[14] J. A. Dix and A. S. Verkman, Crowding Effects on Diffusion in Solutions and Cells, Annu. Rev. Biophys. 37, 247 (2008).

[15] A. V. Weigel, B. Simon, M. M. Tamkun, and D. Krapf, Ergodic and Nonergodic Processes Coexist in the Plasma Membrane as Observed by Single-Molecule Tracking, Proc. Natl. Acad. Sci. U.S.A. 108, 6438 (2011).

[16] T. V. Ratto and M. L. Longo, Anomalous Subdiffusion in Heterogeneous Lipid Bilayers, Langmuir 19, 1788 (2003).

[17] C. Manzo, J. A. Torreno-Pina, P. Massignan, G. J. Lapeyre, Jr., M. Lewenstein, and M. F. Garcia Parajo, Weak Ergodicity Breaking of Receptor Motion in Living Cells Stemming from Random Diffusivity, Phys. Rev. X 5, 011021 (2015).

[18] T. Fujiwara, K. Ritchie, H. Murakoshi, K. Jacobson, and A. Kusumi, Phospholipids Undergo Hop Diffusion in Compartmentalized Cell Membrane, J. Cell Biol. 157, 1071 (2002).

[19] T. Auth and N. S. Gov, Diffusion in a Fluid Membrane with a Flexible Cortical Cytoskeleton, Biophys. J. 96, 818 (2009).

[20] A. V. Weigel, M. M. Tamkun, and D. Krapf, Quantifying the Dynamic Interactions between a Clathrin-Coated Pit and Cargo Molecules, Proc. Natl. Acad. Sci. U.S.A. 110, E4591 (2013).
[21] D. W. Tank, E.-S. Wu, and W. W. Webb, Enhanced Molecular Diffusibility in Muscle Membrane Blebs: Release of Lateral Constraints, J. Cell Biol. 92, 207 (1982).

[22] Y. Lavi, N. Gov, M. Edidin, and L. A. Gheber, Lifetime of Major Histocompatibility Complex Class-I Membrane Clusters Is Controlled by the Actin Cytoskeleton, Biophys. J. 102, 1543 (2012).

[23] M. V. Gudheti, N. M. Curthoys, T. J. Gould, D. Kim, M. S. Gunewardene, K. A. Gabor, J. A. Gosse, C. H. Kim, J. Zimmerberg, and S. T. Hess, Actin Mediates the Nanoscale Membrane Organization of the Clustered Membrane Protein Influenza Hemagglutinin, Biophys. J. 104, 2182 (2013).

[24] M. P. Sheetz, Membrane Skeletal Dynamics: Role in Modulation of Red Cell Deformability, Mobility of Transmembrane Proteins, and Shape, Sem. Hematol. 20, 175 (1983).

[25] M. J. Saxton and K. Jacobson, Single-Particle Tracking: Applications to Membrane Dynamics, Annu. Rev. Biophys. Biomol. Struct. 26, 373 (1997).

[26] K. K. Deal, S. K. England, and M. M. Tamkun, Molecular Physiology of Cardiac Potassium Channels, Physiol. Rev. 76, 49 (1996).

[27] B. B. Mandelbrot and J. W. Van Ness, Fractional Brownian Motions, Fractional Noises and Applications, SIAM Rev. 10, 422 (1968).

[28] W. Deng and E. Barkai, Ergodic Properties of Fractional Brownian-Langevin Motion, Phys. Rev. E 79, 011112 (2009).

[29] M. J. Saxton, Anomalous Diffusion Due to Obstacles: A Monte Carlo Study, Biophys. J. 66, 394 (1994).

[30] D. Ben-Avraham and S. Havlin, Diffusion and Reactions in Fractals and Disordered Systems (Cambridge University Press, Cambridge, England, 2000).

[31] A. V. Weigel, S. Ragi, M. L. Reid, E. K. P. Chong, M. M. Tamkun, and D. Krapf, Obstructed Diffusion Propagator Analysis for Single-Particle Tracking, Phys. Rev. E 85, 041924 (2012).

[32] J. Szymanski and M. Weiss, Elucidating the Origin of Anomalous Diffusion in Crowded Fluids, Phys. Rev. Lett. 103, 038102 (2009).

[33] D. Ernst, M. Hellmann, J. Köhler, and M. Weiss, Fractional Brownian Motion in Crowded Fluids, Soft Matter 8, 4886 (2012).

[34] G. Guigas, C. Kalla, and M. Weiss, The Degree of Macromolecular Crowding in the Cytoplasm and Nucleoplasm of Mammalian Cells Is Conserved, FEBS Lett. 581, 5094 (2007).

[35] M. Weiss, Single-Particle Tracking Data Reveal Anticorrelated Fractional Brownian Motion in Crowded Fluids, Phys. Rev. E 88, 010101 (2013).

[36] H. Scher and E. W. Montroll, Anomalous Transit-Time Dispersion in Amorphous Solids, Phys. Rev. B 12, 2455 (1975).

[37] M. Magdziarz, A. Weron, K. Burnecki, and J. Klafter, Fractional Brownian Motion Versus the Continuous-Time Random Walk: A Simple Test for Subdiffusive Dynamics, Phys. Rev. Lett. 103, 180602 (2009).

[38] G. Rangarajan and M. Ding, First Passage Time Distribution for Anomalous Diffusion, Phys. Lett. A 273, 322 (2000).

[39] V. Tejedor, O. Bénichou, R. Voituriez, R. Jungmann, F. Simmel, C. Selhuber-Unkel, L. B. Oddershede, and 
R. Metzler, Quantitative Analysis of Single Particle Trajectories: Mean Maximal Excursion Method, Biophys. J. 98, 1364 (2010).

[40] M. Weiss, M. Elsner, F. Kartberg, and T. Nilsson, Anomalous Subdiffusion Is a Measure for Cytoplasmic Crowding in Living Cells, Biophys. J. 87, 3518 (2004).

[41] Y. Meroz, I. M. Sokolov, and J. Klafter, Test for Determining a Subdiffusive Model in Ergodic Systems from Single Trajectories, Phys. Rev. Lett. 110, 090601 (2013).

[42] S. Burov, S. M. Ali Tabei, T. Huynh, M. P. Murrell, L. H. Philipson, S. A. Rice, M. L. Gardel, N. F. Scherer, and A. R. Dinner, Distribution of Directional Change as a Signature of Complex Dynamics, Proc. Natl. Acad. Sci. U.S.A. 110, 19689 (2013).

[43] A. M. J. VanDongen, G. C. Frech, J. A. Drewe, R. H. Joho, and A. M. Brown, Alteration and Restoration of $\mathrm{K}^{+}$Channel Function by Deletions at the $\mathrm{N}$-and C-Termini, Neuron 5, 433 (1990).

[44] P. D. Fox, C. J. Haberkorn, A. V. Weigel, J. L. Higgins, E. J. Akin, M. J. Kennedy, D. Krapf, and M. M. Tamkun, Plasma Membrane Domains Enriched in Cortical Endoplasmic Reticulum Function as Membrane Protein Trafficking Hubs, Mol. Biol. Cell 24, 2703 (2013).

[45] P. D. Fox, C. J. Haberkorn, E. J. Akin, P. J. Seel, D. Krapf, and M. M. Tamkun, Induction of Stable ER-PlasmaMembrane Junctions by Kv2.1 Potassium Channels, J. Cell Sci. 128, 2096 (2015).

[46] See Supplemental Material at http://link.aps.org/ supplemental/10.1103/PhysRevX.7.011031 for a superresolution video of the cortical actin and details about experimental methods and numerical simulations.

[47] A. Tsuji and S. Ohnishi, Restriction of the Lateral Motion of Band 3 in the Erythrocyte Membrane by the Cytoskeletal Network: Dependence on Spectrin Association State, Biochemistry 25, 6133 (1986).

[48] M. Edidin, M. C. Zuniga, and M. P. Sheetz, Truncation Mutants Define and Locate Cytoplasmic Barriers to Lateral Mobility of Membrane Glycoproteins, Proc. Natl. Acad. Sci. U.S.A. 91, 3378 (1994).

[49] N. L. Andrews, K. A. Lidke, J. R. Pfeiffer, A. R. Burns, B. S. Wilson, J. M. Oliver, and D. S. Lidke, Actin Restricts FceRI Diffusion and Facilitates Antigen-Induced Receptor Immobilization, Nat. Cell Biol. 10, 955 (2008).

[50] J. Wiedenmann, S. Ivanchenko, F. Oswald, F. Schmitt, C. Röcker, A. Salih, K.-D. Spindler, and G. Nienhaus, EosFP, A Fluorescent Marker Protein with UV-Inducible Green-to-Red Fluorescence Conversion, Proc. Natl. Acad. Sci. U.S.A. 101, 15905 (2004).

[51] I. Izeddin, C. G. Specht, M. Lelek, X. Darzacq, A. Triller, C. Zimmer, and M. Dahan, Super-Resolution Dynamic Imaging of Dendritic Spines Using a Low-Affinity Photoconvertible Actin Probe, PLoS One 6, e15611 (2011).

[52] J. Riedl, A. H. Crevenna, K. Kessenbrock, J. Haochen Yu, D. Neukirchen, M. Bista, F. Bradke, D. Jenne, T. A. Holak, Z. Werb et al., Lifeact: A Versatile Marker to Visualize F-actin, Nat. Methods 5, 605 (2008).

[53] B. Treanor, D. Depoil, A. Gonzalez-Granja, P. Barral, M. Weber, O. Dushek, A. Bruckbauer, and F. D. Batista, The Membrane Skeleton Controls Diffusion Dynamics and
Signaling through the B Cell Receptor, Immunity 32, 187 (2010).

[54] J. A. Torreno-Pina, C. Manzo, M. Salio, M. C. Aichinger, A. Oddone, M. Lakadamyali, D. Shepherd, G. S. Besra, V. Cerundolo, and M. F. Garcia-Parajo, The Actin Cytoskeleton Modulates the Activation of iNKT Cells by Segregating CDld Nanoclusters on Antigen-Presenting Cells, Proc. Natl. Acad. Sci. U.S.A. 113, E772 (2016).

[55] M. J. Rust, M. Bates, and X. Zhuang, Sub-diffraction-Limit Imaging by Stochastic Optical Reconstruction Microscopy (STORM), Nat. Methods 3, 793 (2006).

[56] G. T. Dempsey, J. C. Vaughan, K. H. Chen, M. Bates, and X. Zhuang, Evaluation of Fluorophores for Optimal Performance in Localization-Based Super-Resolution Imaging, Nat. Methods 8, 1027 (2011).

[57] K. Xu, H. P. Babcock, and X. Zhuang, Dual-Objective STORM Reveals Three-Dimensional Filament Organization in the Actin Cytoskeleton, Nat. Methods 9, 185 (2012).

[58] F. Meyer and S. Beucher, Morphological Segmentation, J. Visual Commun. Image Represent 1, 21 (1990).

[59] C. Klüppelberg, Subexponential Distributions and Integrated Tails, J. Appl. Probab. 25, 132 (1988).

[60] A. N. Kolmogorov, On the Logarithmically Normal Law of Distribution of the Size of Particles Under Pulverisation, Dokl. Akad. Nauk SSSR 31, 99 (1941).

[61] B. Epstein, The Mathematical Description of Certain Breakage Mechanisms Leading to the Logarithmic-Normal Distribution, J. Franklin Inst. 244, 471 (1947).

[62] B. B. Mandelbrot, The Fractal Geometry of Nature (Macmillan, London, 1983), Vol. 173.

[63] S. Lovejoy, Area-Perimeter Relation for Rain and Cloud Areas, Science 216, 185 (1982).

[64] A. R. Imre and J. Bogaert, The Fractal Dimension as a Measure of the Quality of Habitats, Acta Biotheoretica 52, 41 (2004).

[65] P. S. Addison, Fractals and Chaos: An Illustrated Course (Institute of Physics Publishing, Bristol, UK, 1997).

[66] W. I. Newman, D. L. Turcotte, and A. M. Gabrielov, Fractal Trees with Side Branching, Fractals 05, 603 (1997).

[67] R. E. Horton, Erosional Development of Streams and Their Drainage Basins; Hydrophysical Approach to Quantitative Morphology, Geol. Soc. Am. Bull. 56, 275 (1945).

[68] D. Pantaloni, R. Boujemaa, D. Didry, P. Gounon, and M.-F. Carlier, The Arp2/3 Complex Branches Filament Barbed Ends: Functional Antagonism with Capping Proteins, Nat. Cell Biol. 2, 385 (2000).

[69] K. Gowrishankar, S. Ghosh, S. Saha, C. Rumamol, S. Mayor, and M. Rao, Active Remodeling of Cortical Actin Regulates Spatiotemporal Organization of Cell Surface Molecules, Cell 149, 1353 (2012).

[70] K. M. S. O'Connell, A. S. Rolig, J. D. Whitesell, and M. M. Tamkun, Kv2.1 Potassium Channels Are Retained within Dynamic Cell Surface Microdomains that Are Defined by a Perimeter Fence, J. Neurosci. 26, 9609 (2006).

[71] K. Jaqaman, D. Loerke, M. Mettlen, H. Kuwata, S. Grinstein, S. L. Schmid, and G. Danuser, Robust Single-Particle Tracking in Live-Cell Time-Lapse Sequences, Nat. Methods 5, 695 (2008). 